\title{
$\beta$-Adrenergic receptors in the insular cortex are differentially involved in aversive vs. incidental context memory formation
}

\author{
María Isabel Miranda, ${ }^{1,3}$ Elizabeth Sabath, ${ }^{1}$ Luis Nuñez-Jaramillo, ${ }^{1,2}$ \\ and Liliana Purón-Sierra ${ }^{1}$ \\ ${ }^{1}$ Departamento de Neurobiología Conductual y Cognitiva, Instituto de Neurobiología, Universidad Nacional Autónoma de México, \\ Campus Juriquilla, Querétaro, 96230 Mexico; ${ }^{2}$ División de Ciencias de la Salud, Universidad de Quintana Roo, Chetumal, Quintana \\ Roo, 77019 Mexico
}

\begin{abstract}
The goal of this research was to determine the effects of $\beta$-adrenergic antagonism in the IC before or after inhibitory avoidance (IA) training or context pre-exposure in a latent inhibition protocol. Pretraining intra-IC infusion of the $\beta$-adrenergic antagonist propranolol disrupted subsequent IA retention and impaired latent inhibition of IA, but had no effect on formation of memory for an inert context (termed incidental memory). These results indicate that IC $\beta$-adrenergic receptors are necessary for memory acquisition of an aversive, but not an inconsequential, context. Nevertheless, subsequent association of a familiar and hitherto inconsequential context with an unconditioned stimulus (US) does require activation of these receptors during its initial acquisition.
\end{abstract}

Substantial evidence indicates that long-term memories related to emotional experiences are particularly robust (Buchanan 2007). However, a substantial component of our memories are related to incidental stimuli, that is, stimuli that have no overt emotional relation. Recent data have implicated the insular cortex (IC) in the formation of such "incidental" memories (BermudezRattoni 2004; Miranda and Bermudez-Rattoni 2007; Miranda et al. 2008). The IC has substantial connections with the limbic system and the "reward circuit," and receives information from all sensory modalities (Mesulam and Mufson 1982; Mufson and Mesulam 1982). Functionally, the IC has been demonstrated to play a critical role in memory performance in a variety of memory tasks that use an aversive unconditioned stimulus (US), including taste recognition memory (Bermudez-Rattoni 2004), Morris spatial water maze (Bermudez-Rattoni and McGaugh 1991; Gutierrez et al. 1999), and the inhibitory-avoidance (IA) task (Bermudez-Rattoni and McGaugh 1991; Bermudez-Rattoni et al. 1991), as well as memory performance in a task with no overtly aversive stimuli, namely, object recognition (Bermudez-Rattoni et al. 2005).

Though the IC has been shown to be necessary for spatial and avoidance memory formation (Bermudez-Rattoni and McGaugh $1991)$ and the $\beta$-adrenergic system has been shown to play a critical role in IA consolidation (Barros et al. 2001; Lalumiere et al. 2004; McGaugh 2004), the functions of $\beta$-adrenergic activity in the IC during IA acquisition, consolidation, and retrieval are unknown. Hence, the present study was designed to examine the involvement of $\beta$-adrenergic receptors in the IC during acquisition and memory consolidation of the IA task. We further investigated whether $\beta$-adrenergic receptors in the IC might be differentially involved in mediating aversive and incidental contextual memory formation, as well as their role in a latent inhibition variant of the IA task. Specifically, the latent inhibition IA

\footnotetext{
${ }^{3}$ Corresponding author.
}

E-mail mirandami@unam.mx.

Article is online at http://www.learnmem.org/cgi/doi/10.1101/lm.2126111. paradigm involves pre-exposing animals to the IA dark compartment in the absence of a footshock and then assessing their latency to enter that same dark compartment the following day, at which time a footshock is then administered. Lubow (1989) showed that this kind of pre-exposure reduces the probability of the apparatus context being associated with a footshock applied when the animal is placed in that context on a subsequent day-a phenomenon known as latent inhibition.

All methods used complied with the Rules in Health Matters (Ministry of Health, Mexico) and were approved by the local Animal Care and Use Committee. One hundred seventy-eight male, Sprague-Dawley rats (250-280 g at the time of arrival) were used. Animals were housed individually under a standard 12-h light/12-h dark cycle at $22^{\circ} \mathrm{C}$ with food and water available ad libitum. IA acquisition and retrieval were performed during the dark phase of the light cycle. For surgery, the rats were anesthetized with ketamine $(6 \mathrm{mg} / \mathrm{kg})$ and xylazine $(70 \mathrm{mg} / \mathrm{kg})$ and implanted bilaterally via stereotaxic frame (Kopf Instruments) with guide cannulae (23 gauge, $15 \mathrm{~mm}$ ) aimed at the left and right IC (coordinates: anteroposterior, $1.2 \mathrm{~mm}$; mediolateral, $\pm 5.5 \mathrm{~mm}$; dorsoventral, $-3.0 \mathrm{~mm}$ from Bregma) (Paxinos and Watson 1998; Fig. 1).

The animals were allowed to recover from surgery for $7 \mathrm{~d}$. Then, to improve discrimination between the IA compartments, they were handled and habituated to the light compartment (LC) of the IA apparatus for 4 min daily for $2 \mathrm{~d}$. Briefly, the IA apparatus was a trough-shaped alley (previously described by Miranda and Bermudez-Rattoni 2007). One compartment, the LC (31-cm long), was brightly lit, while the other, the dark compartment (60-cm long), where the footshock is applied, was dimly lit. The two compartments were distinctly separated by an acrylic divider with a small hole $(6.5-\mathrm{cm}$ diameter) through which the rat passes to enter the dark compartment. Twenty-four hours after habituation, the animals designated for IA training were habituated for an additional day as described above, and then trained using a classic single-trial IA procedure on the following day (see Fig. 2, protocol diagrams). For IA training, each animal was placed 


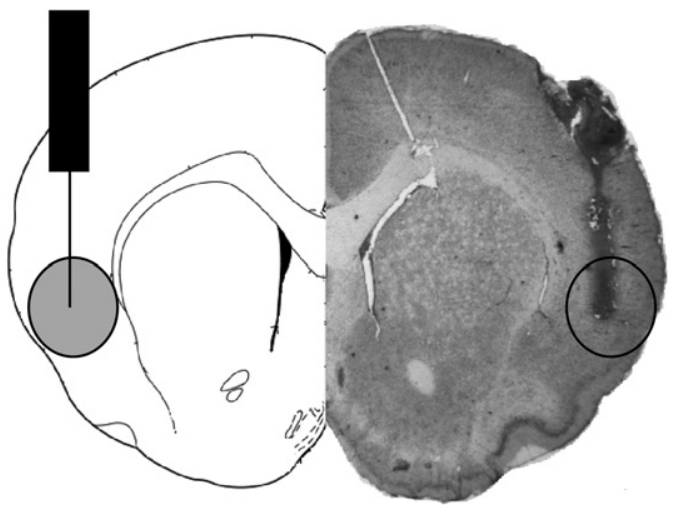

Figure 1. Sketch (left) and microphotography of the IC depicting the injection site.

in the LC, and the latency for it to enter the dark compartment was recorded. After the animal entered the dark compartment, the sliding divider was advanced to close the passageway, and the rat received an inescapable footshock $(0.29 \mathrm{~mA}, 1.0 \mathrm{sec}) ; 15$ sec later, the rat was removed from the dark compartment. For IA retrieval testing, each rat was placed in the LC $48 \mathrm{~h}$ after training, and its latency to enter the dark compartment (maximum of $600 \mathrm{sec}$ ) was recorded and taken as a measure of memory retention; no footshock was delivered in the retrieval test.

Animals designated for the latent inhibition experiment were divided into non-pre-exposed control and pre-exposed groups. One day after habituation, animals in the control (non-pre-exposed) groups were placed in the LC with no access to the dark compartment for $4 \mathrm{~min}$. Meanwhile, animals in the pre-exposed groups were placed in the LC of the IA apparatus with access to the dark compartment for $4 \mathrm{~min}$ without receiving any footshocks; each rat's latency to enter the dark compartment on the pre-exposure day was recorded. Note that rats in the non-pre-exposed groups were treated identically to pre-exposed rats (handling and novel context exposure on the pre-exposure day) with the exception of exposure to the dark compartment that was to become the shock context during later training. Rats in both the control and pre-exposed groups were then subjected to IA training $24 \mathrm{~h}$ later, as described previously (Fig. 3, protocol diagrams). A decrease in the rats' latencies to enter the dark compartment from the pre-exposure trial to the training trial was taken as evidence of incidental context memory the next day during IA acquisition. In rats pre-exposed to the dark compartment, reduced avoidance of the shock context in the form of shorter latencies to re-enter the dark compartment on the test day, relative to rats non-pre-exposed to the dark compartment, was interpreted as a deficit in context-shock association and, thus, as evidence of latent inhibition.

Propranolol $(1 \mu \mathrm{g} / 0.5 \mu \mathrm{L}$, SIGMA, dissolved in isotonic saline) or saline was infused bilaterally into the IC through 30-gauge injection needles connected to a $10-\mu \mathrm{L}$ Hamilton microsyringe by polyethylene tubing. Using an automated syringe pump (Sage Instruments), $0.5 \mu \mathrm{L}$ of solution was infused over $60 \mathrm{sec}$, and the injection needle was kept in place for an additional $60 \mathrm{sec}$. The propranolol dose $(1 \mu \mathrm{g} / 0.5 \mu \mathrm{L})$ was selected on the basis of previous findings (Miranda et al. 2008).

To test the effects of $\beta$-adrenergic antagonism on IA acquisition and consolidation, infusions were delivered $15 \mathrm{~min}$ before IA training (BT groups, Fig. 2A) or immediately after IA training (AT groups, Fig. 2B), respectively. To test the effects of $\beta$-adrenergic antagonism on latent inhibition, infusions were delivered $15 \mathrm{~min}$ before the pre-exposure trial (Fig. 3A). The control (non-pre-exposed) groups of animals received infusions before or after their 4-min period in the LC with the passageway closed (Fig. 3B).

At the end of behavioral experiments, the rats were anesthetized with an overdose of sodium pentobarbital $(\approx 115 \mathrm{mg} / \mathrm{kg})$ and perfused intracardially with $0.9 \%$ saline (wt/vol). The brains were placed in $10 \%$ formaldehyde for $4 \mathrm{~d}$ and then in a $30 \%$ sucrose solution for cryoprotection. Fifty-micron-thick sections were stained with cresyl violet and then examined under a light microscope to determine the location of the cannula tips within the IC (Fig. 1). The results of each experiment were analyzed by means of a factorial analysis of variance (ANOVA), followed by Fisher's post-hoc tests to determine the sources of the significance.

The effects of intra-IC propranolol infusion on acquisition and/or consolidation of IA are shown in Figure 2. The latency to enter the dark compartment during the training trial for the propranolol BT group $(23.0 \pm 2.8 \mathrm{sec})$ was similar to that for the saline BT group ( $22.3 \pm 1.5 \mathrm{sec}$; ANOVA $P>0.05$ vs. propranolol BT), indicating that pretraining propranolol infusion into the IC did not affect motor or motivational responses during IA training (Fig. 2A). The propranolol BT group did, however, exhibit significantly shorter latencies to enter the dark compartment at the retention test $(235.8 \pm 58.5 \mathrm{sec})$ relative to those exhibited by the saline BT group $\left(516.7 \pm 56.5 \mathrm{sec}\right.$; factorial ANOVA $F_{(1,23)}=$ $10.81, P<0.01)$, indicating that pretraining, intra-IC propranolol infusions impaired subsequent IA retention (Fig. 2A). A significant effect of propranolol on retention was not observed when infusions were made $15 \mathrm{~min}$ after training (saline AT $=335.89 \pm$ $73.4 \mathrm{sec}$, propranolol AT $=283.4 \pm 69.9 \mathrm{sec}, P>0.05$ ) (Fig. $2 \mathrm{~B}$ ).

The effects of intra-IC propranolol infusion, before (BP) or after (AP) pre-exposure, are shown in Figure 3. A factorial ANOVA test revealed significant variability among the groups in terms of their latencies to enter the shock compartment during the training trial in the latent inhibition experiment $\left(F_{(3,48)}=\right.$ 8.09, $P<0.01$ ) (Fig. 3A, left graph). Specifically, in the absence of drugs, the context pre-exposed, saline BP-infused rats had shorter latencies to enter the dark compartment $1 \mathrm{~d}$ after context pre-exposure than did the non-pre-exposed, saline BP-infused rats $(P<0.001)$. Thus, pre-exposure to the dark compartment (without a footshock, US) reduced rats' latencies to enter this compartment the next day during IA training. This reduced latency provides evidence for the presence of memory for the context, as rats are less inhibited to enter familiar places relative to novel places (Roesler et al. 2005; Miranda and Bermudez-Rattoni 2007). And as shown in the right graph of Figure 3A, this effect of context pre-exposure persisted in rats that had been infused with propranolol before context pre-exposure $(P<0.05$, non-preexposed vs. pre-exposed propranolol groups, Fisher's post hoc test), indicating that the propranolol treatment did not significantly affect memory for the context pre-exposure. This memory for the context from the pre-exposure day can be considered an incidental memory since the context had not yet been associated with any US at the time of entry during this IA training, as no shocks (or any other aversive or rewarding stimulation) had yet been delivered. Thus, incidental memories are memories for inconsequential stimuli. Following subsequent delivery of the footshock in the dark compartment during IA training, the dark compartment context then becomes a complex conditioned stimulus (CS) associated with a footshock, an aversive US.

At the IA retention test, $1 \mathrm{~d}$ after IA training and $2 \mathrm{~d}$ after preexposure (protocol illustrated in Fig. 3A, above graphs), significant differences in entrance latency were observed between the experimental groups $\left(F_{(3,48)}=5.969, P<0.01\right)$ (Fig. 3A, right graph). The Fisher post hoc test revealed that the pre-exposed saline BP group had a significantly lower mean latency to enter the 

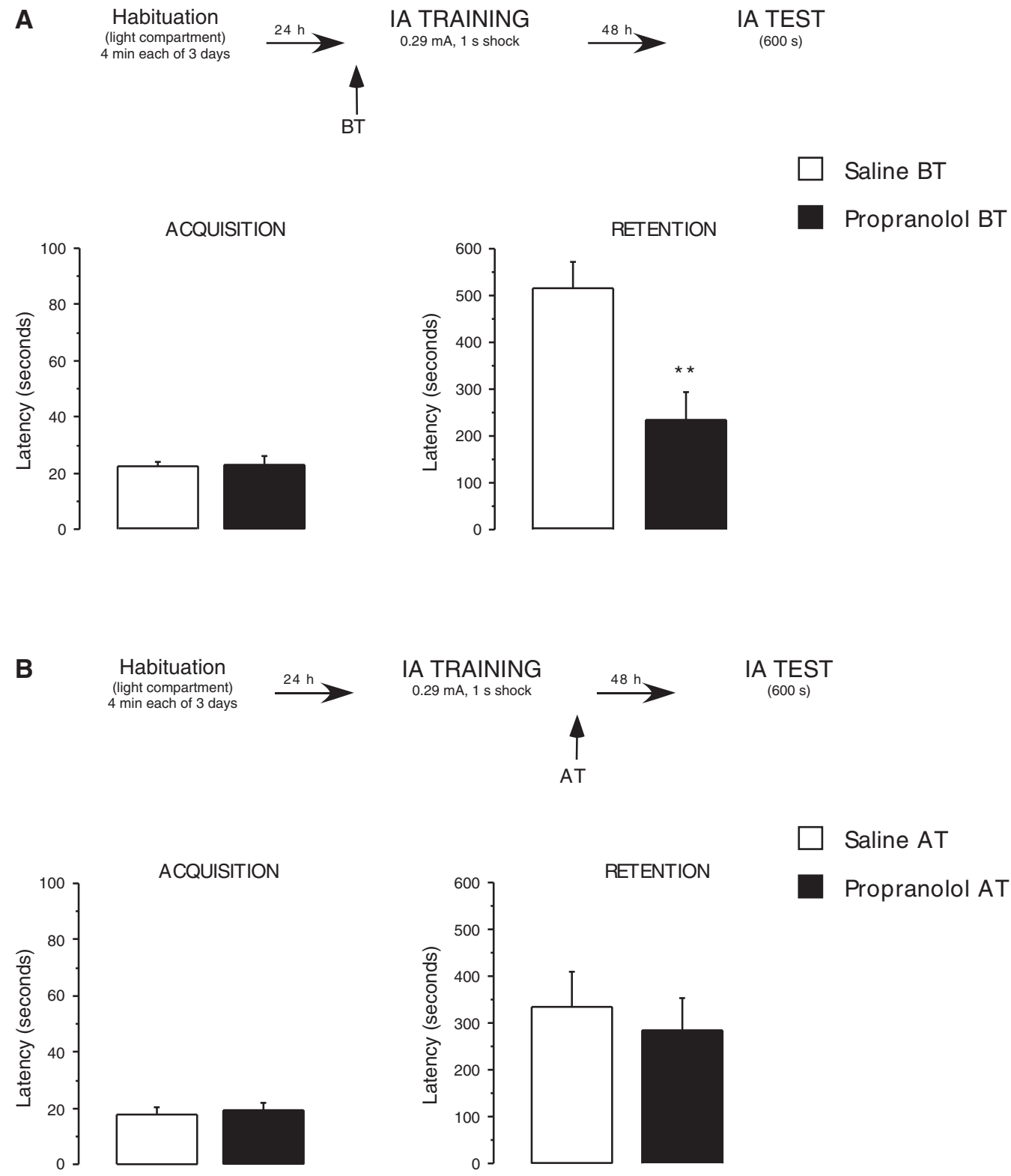

Figure 2. Intra-IC propranolol infusion disrupts IA acquisition and/or consolidation when administered before training $(A)($ saline $B T, n=10$; propranolol BT, $n=15$ ), but does not disrupt consolidation when administered after training (B) (saline AT, $n=9$, propranolol AT, $n=14$ ). The arrows in the protocol diagrams (above the graphs) indicate the time of infusion in each experiment. Reported data are mean latencies to enter the dark compartment \pm SEM. $(* *) P<0.01$ vs. saline control group.

dark compartment than the non-pre-exposed saline BP group $(P<$ 0.01), demonstrating a latent inhibition effect-that is, an inhibitory effect of dark compartment pre-exposure on the subsequent CS-US (i.e., context-footshock) association established during IA training. As shown in the right panel of Figure 3A, this latent inhibition effect on IA retention was notably absent in rats given intra-IC infusions of the $\beta$-adrenergic antagonist propranolol before the pre-exposure session $(P>0.05$, pre-exposed propranolol BP vs. non-pre-exposed propranolol BP) (Fig. 3A). This result shows that propranolol infusions before context pre-exposure disrupted the development of latent inhibition, since animals in the pre-exposed propranolol BP group avoided the dark compartment to the same extent as animals who had not been subjected to pre-exposure.
Similar to our observations with BP groups, when intra-IC propranolol infusions were administered after pre-exposure in the AP groups (Fig. 3B, left graph), a factorial ANOVA showed significant group differences in mean entrance latencies during IA training $\left(F_{(3,74)}=11.6, P<0.0001\right)$. A Fisher post hoc test revealed differences between the pre-exposed and non-pre-exposed saline AP groups $(P<0.001)$, as well as between the pre-exposed and non-pre-exposed propranolol AP groups $(P<0.01)$. Thus, propranolol infusions after pre-exposure to the dark compartment did not disrupt incidental context memory consolidation, since preexposed animals showed reduced latency to enter the dark compartment during IA training regardless of whether they had received a drug or saline vehicle following the pre-exposure trial. A factorial ANOVA test also showed significant group differences 


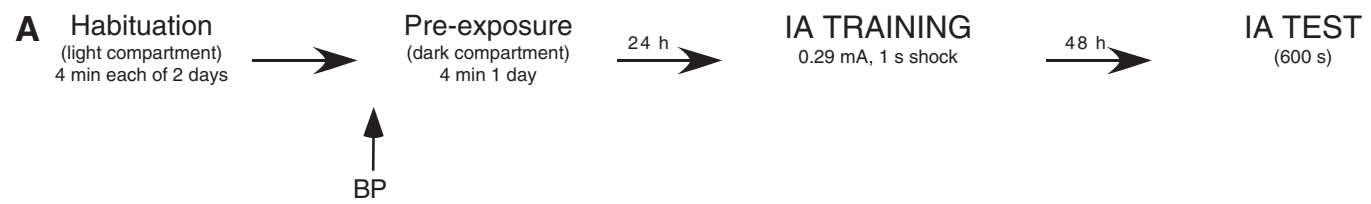

ACQUISITION DURING IA TRAINING TRIAL
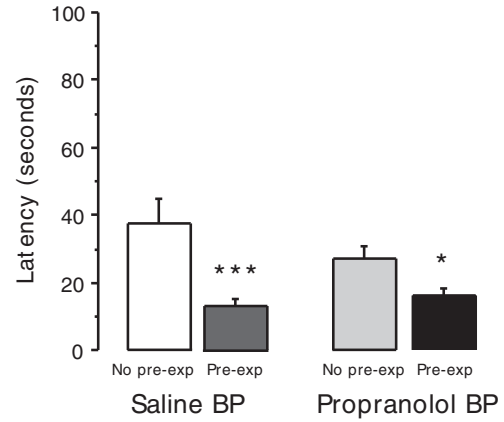

POST-TRAINING IA RETENTION TEST

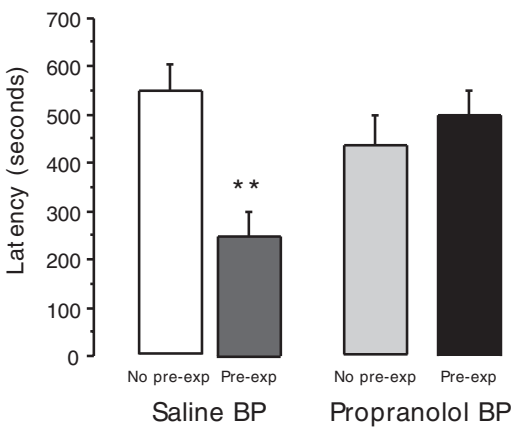

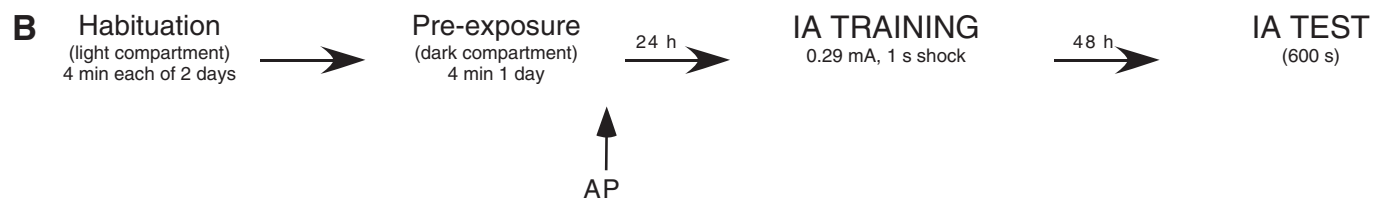

ACQUISITION DURING IA TRAINING TRIAL

POST-TRAINING IA RETENTION TEST
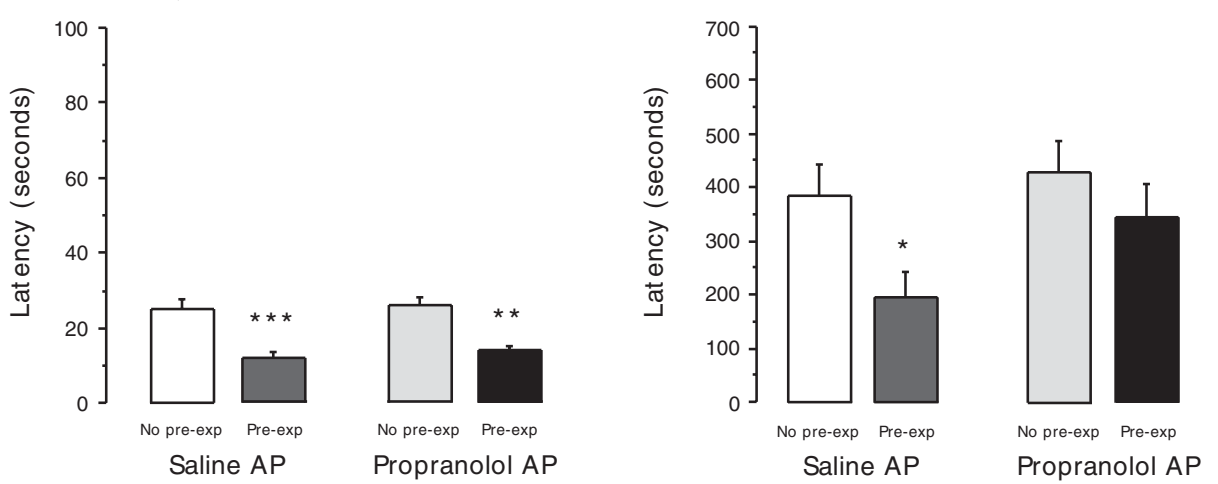

Figure 3. Intra-IC propranolol infusion disrupts latent inhibition of the IA task whether administered before ( $A$ ) (no pre-exposure saline BP, $n=11 ;$ no pre-exposure propranolol BP, $n=12$; pre-exposure saline BP, $n=14$; pre-exposure propranolol BP, $n=15$ ) or after (B) (no pre-exposure saline AP, $n=21$; no pre-exposure propranolol AP, $n=17$; pre-exposure saline AP, $n=21$; pre-exposure propranolol AP, $n=19$ ) the pre-exposure session. The arrows in the protocol diagrams (above the graphs) indicate the time of infusion in each experiment. $\left(^{*}\right) P<0.05,\left({ }^{* *}\right) P<0.01,(* * *) P<0.0001$ vs. no pre-exposure group given the same infusion substance at the same experimental time point.

$\left(F_{(3,74)}=3.402, P<0.05\right)$ (Fig. 3B, right graph). A Fisher post hoc test revealed a significant difference in mean entrance latency between pre-exposed and non-pre-exposed saline AP groups $(P<0.05)$, replicating the latent inhibition effect observed in the BP groups reported above. Meanwhile, the mean entrance latency of the pre-exposed propranolol AP group did not differ from that of the non-pre-exposed propranolol AP group (Fig. 3B). Thus, propranolol infusions administered after context pre-exposure disrupted latent inhibition in a manner similar to that observed when infusions were administered prior to context pre-exposure.

In summary, the present results demonstrate that $\beta$-adrenergic receptors in the IC play a critical role during IA acquisition since intra-IC propranolol infusions prior to IA training disrupted IA retention, while infusions immediately after training did not significantly affect subsequent IA retention. The finding that intra-IC propranolol affected IA retention when it was administered before, but not after, IA training indicates that $\beta$-adrenergic receptor activation is important for the acquisition, but perhaps not the consolidation, of memory for the IA task. It should be noted that intra-IC propranolol administration before IA training did not affect entrance latency or footshock reaction (data not shown) during training, relative to control salineinfused animals, indicating that the drug treatment did not alter perception, motivation, or locomotion. These findings show that $\beta$-adrenergic activity in the IC is necessary during aversive 
context learning, in agreement with substantial evidence showing that $\beta$-adrenergic receptor antagonism selectively reduces memory formation related aversive or emotional events (McGaugh and Roozendaal 2002; Ramos and Arnsten 2007), and that enhanced memory associated with emotional experiences involves activation of the $\beta$-adrenergic system (for review, see McGaugh 2000).

A second interesting finding reported here is the differential role of IC $\beta$-adrenergic receptors in incidental context memory formation vs. their role in latent inhibition. That is, antagonism of $\beta$-adrenergic receptors on the pre-exposure day (either before or after the pre-exposure) did not disrupt acquisition or consolidation of the incidental context memory as assessed by mean entrance latency to enter the dark compartment during IA training ( $1 \mathrm{~d}$ after the pre-exposure trial). However, these infusions did significantly reduce development of latent inhibition of IA, since the same animals treated with the $\beta$-adrenoreceptor blocker on the pre-exposure day had high entrance latencies similar to those of non-pre-exposed control subjects during the IA retention test. The fact that $\beta$-blockade in the IC affected only latent inhibition, while leaving incidental memory formation intact, is relevant to the theory that latent inhibition reflects a "mechanism" that interferes with the full processing of a previously encoded stimulus that was inert at the time it was first presented (De la Casa and Lubow 1995).

Based on the present findings, we posit that IC $\beta$-adrenergic receptors play a critical role in the classical acquisition of CS-US association (as during IA training) (see Fig. 2) as well as in a parallel process required to relate a previously inconsequential stimulus (i.e., context during pre-exposure) to a US (i.e., aversive footshock during conditioning), but are not necessary for the formation of an incidental memory (familiarization) of the inconsequential stimulus (i.e., dark compartment context) before it is paired with a US (i.e., footshock). This interpretation of our observations is consistent with a body of evidence suggesting that the IC contributes to a multibrain region complex activity that produces contrasting effects during different stages of stimulus pre-exposure (Roesler et al. 2005; Malin and McGaugh 2006; Miranda and Bermudez-Rattoni 2007; Barak and Weiner 2010). Hence, in accordance with previous evidence, we infer that intra-IC propranolol infusions in the present experiments affected the capacity to subsequently process information acquired during a prior inconsequential experience, in a manner that disrupted the mechanism needed to respond to the changed stimulus properties during conditioning, namely its new association with a US. This interpretation is in line with evidence that certain structures are important for altering responses based upon changes in stimulus-consequence associations, an aspect of cognitive flexibility (Hatfield et al. 1996; Malkova et al. 1997; Baxter et al. 2000; Cooper and Mizumori 2001; Schoenbaum et al. 2003; Malin and McGaugh 2006; Barak and Weiner 2010).

Though the noradrenergic system has been implicated as a central player in controlling attention (Aston-Jones et al. 1985) as well as in inhibiting attention to irrelevant stimuli (Mason and Iversen 1979), abundant evidence indicates that central noradrenergic depletion has no effect on latent inhibition, particularly when using the conventional latent inhibition paradigm used here (i.e., single-stimulus pre-exposure followed by conditioning with the same stimulus) (Archer 1982; Robbins 1984; Tsaltas et al. 1984; Archer et al. 1985). Thus, as suggested by Weiner (1990) it seems that noradrenergic activity may play an important role in attention to inconsequential experiences (preexposure) "only when optimal processing capacity is required for certain input in comparison to other inputs, particularly if such a discrimination occurs under complex or stressful conditions" (see also Robbins 1984; Oades et al. 1985; Sara 2009).
In conclusion, the present experiments together demonstrate that $\beta$-adrenergic receptors in the IC play differential roles during aversive contextual memory formation. Specifically, in addition to enabling the formation of CS-US associations in classical conditioning paradigms (e.g., IA), $\beta$-adrenergic activity appears to also enable the development of latent inhibition, in which initially inconsequential unpaired stimuli are inhibited from later gaining an association with a US (e.g., IA training after context pre-exposure). They further indicate that $\beta$-adrenergic receptor involvement in the acquisition and/or consolidation of a presumably non-emotional incidental memory of an inconsequential context are critical for the ability to form associations between that context and USs paired with it at a later time point, though the initial formation of memory for the context itself (familiarity), independent of associating it with a US, appears not to require noradrenergic involvement in the IC.

\section{Acknowledgments}

This research was supported by PAPIIT IN201308 and CONACyT C54524. We thank Ann Power, Dorothy Pless, and Shaun Harris for the English revision of the final text. We also thank Alejandro Rangel, Martín García, Ángela Gabriela Vera-Rivera, Nydia Hernández-Ríos, and Lorena López for their technical assistance.

\section{References}

Archer T. 1982. The role of noradrenaline in learned behaviors: Studies using DSP4. Scand J Psychol Suppl 1: 61-71.

Archer T, Jarbe TU, Mohammed AK, Priedite G. 1985. The effect of stimulus preexposure upon the context effect in taste-aversion learning in noradrenaline-depleted rats. Scand J Psychol 26: 158-169.

Aston-Jones G, Shaver R, Dinan TG. 1985. Nucleus basalis neurons exhibit axonal branching with decreased impulse conduction velocity in rat cerebrocortex. Brain Res 325: 271-285.

Barak S, Weiner I. 2010. Differential role of muscarinic transmission within the entorhinal cortex and basolateral amygdala in the processing of irrelevant stimuli. Neuropsychopharmacology 35: 1073-1082.

Barros DM, Mello e Souza T, De David T, Choi H, Aguzzoli A, Madche C, Ardenghi P, Medina JH, Izquierdo I. 2001. Simultaneous modulation of retrieval by dopaminergic $\mathrm{D}(1), \beta$-noradrenergic, serotonergic-1A and cholinergic muscarinic receptors in cortical structures of the rat. Behav Brain Res 124: 1-7.

Baxter MG, Parker A, Lindner CC, Izquierdo AD, Murray EA. 2000. Control of response selection by reinforcer value requires interaction of amygdala and orbital prefrontal cortex. J Neurosci 20: 4311-4319.

Bermudez-Rattoni F. 2004. Molecular mechanisms of taste-recognition memory. Nat Rev Neurosci 5: 209-217.

Bermudez-Rattoni F, McGaugh JL. 1991. Insular cortex and amygdala lesions differentially affect acquisition on inhibitory avoidance and conditioned taste aversion. Brain Res 549: 165-170.

Bermudez-Rattoni F, Introini-Collison IB, McGaugh JL. 1991. Reversible inactivation of the insular cortex by tetrodotoxin produces retrograde and anterograde amnesia for inhibitory avoidance and spatial learning. Proc Natl Acad Sci 88: 5379-5382.

Bermudez-Rattoni F, Okuda S, Roozendaal B, McGaugh JL. 2005. Insular cortex is involved in consolidation of object recognition memory. Learn Mem 12: 447-449.

Buchanan TW. 2007. Retrieval of emotional memories. Psychol Bull 133: $761-779$.

Cooper BG, Mizumori SJ. 2001. Temporary inactivation of the retrosplenial cortex causes a transient reorganization of spatial coding in the hippocampus. J Neurosci 21: 3986-4001.

De la Casa G, Lubow RE. 1995. Latent inhibition in conditioned taste aversion: The roles of stimulus frequency and duration and the amount of fluid ingested during preexposure. Neurobiol Learn Mem 64: $125-132$.

Gutierrez H, Hernandez-Echeagaray E, Ramirez-Amaya V, Bermudez-Rattoni F. 1999. Blockade of N-methyl-D-aspartate receptors in the insular cortex disrupts taste aversion and spatial memory formation. Neuroscience 89: 751-758.

Hatfield T, Han JS, Conley M, Gallagher M, Holland P. 1996. Neurotoxic lesions of basolateral, but not central, amygdala interfere with Pavlovian second-order conditioning and reinforcer devaluation effects. J Neurosci 16: 5256-5265. 
Lalumiere RT, Nguyen LT, McGaugh JL. 2004. Post-training intrabasolateral amygdala infusions of dopamine modulate consolidation of inhibitory avoidance memory: Involvement of noradrenergic and cholinergic systems. Eur J Neurosci 20: 2804-2810.

Lubow RE. 1989. Latent inhibition and conditioned attention theory. Cambridge University Press, New York.

Malin EL, McGaugh JL. 2006. Differential involvement of the hippocampus, anterior cingulate cortex, and basolateral amygdala in memory for context and footshock. Proc Natl Acad Sci 103: 1959-1963.

Malkova L, Gaffan D, Murray EA. 1997. Excitotoxic lesions of the amygdala fail to produce impairment in visual learning for auditory secondary reinforcement but interfere with reinforcer devaluation effects in rhesus monkeys. I Neurosci 17: 6011-6020.

Mason ST, Iversen SD. 1979. Theories of the dorsal bundle extinction effect. Brain Res 180: 107-137.

McGaugh JL. 2000. Memory-a century of consolidation. Science 287: $248-251$.

McGaugh JL. 2004. The amygdala modulates the consolidation of memories of emotionally arousing experiences. Annu Rev Neurosci 27: $1-28$.

McGaugh JL, Roozendaal B. 2002. Role of adrenal stress hormones in forming lasting memories in the brain. Curr Opin Neurobiol 12: 205-210.

Mesulam MM, Mufson EJ. 1982. Insula of the old world monkey. III: Efferent cortical output and comments on function. J Comp Neurol 212: $38-52$.

Miranda MI, Bermudez-Rattoni F. 2007. Cholinergic activity in the insular cortex is necessary for acquisition and consolidation of contextual memory. Neurobiol Learn Mem 87: 343-351.

Miranda MI, Rodriguez-Garcia G, Reyes-Lopez JV, Ferry B, Ferreira G. 2008. Differential effects of $\beta$-adrenergic receptor blockade in basolateral amygdala or insular cortex on incidental and associative taste learning. Neurobiol Learn Mem 90: 54-61.

Mufson EJ, Mesulam MM. 1982. Insula of the old world monkey. II: Afferent cortical input and comments on the claustrum. J Comp Neurol 212: $23-37$.

Oades R, Taghzouti K, Simon H, Le Moal M. 1985. Dopamine-sensitive alternation and collateral behaviour in a Y-maze: Effects of damphetamine and haloperidol. Psychopharmacology (Berl) 85: 123-128.

Paxinos G, Watson C. 1998. The rat brain in stereotaxic coordinates. Academic Press, San Diego, CA.

Ramos BP, Arnsten AF. 2007. Adrenergic pharmacology and cognition: Focus on the prefrontal cortex. Pharmacol Ther 113: 523-536.

Robbins TW. 1984. Cortical noradrenaline, attention and arousal. Psychol Med 14: 13-21.

Roesler R, Reolon GK, Luft T, Martins MR, Schroder N, Vianna MR, Quevedo J. 2005. NMDA receptors mediate consolidation of contextual memory in the hippocampus after context preexposure. Neurochem Res 30: $1407-1411$.

Sara SJ. 2009. The locus coeruleus and noradrenergic modulation of cognition. Nat Rev Neurosci 10: 211-223.

Schoenbaum G, Setlow B, Saddoris MP, Gallagher M. 2003. Encoding predicted outcome and acquired value in orbitofrontal cortex during cue sampling depends upon input from basolateral amygdala. Neuron 39: $855-867$.

Tsaltas E, Preston GC, Rawlins JN, Winocur G, Gray JA. 1984. Dorsal bundle lesions do not affect latent inhibition of conditioned suppression. Psychopharmacology (Berl) 84: 549-555.

Weiner I. 1990. Neural substrates of latent inhibition: The switching model. Psychol Bull 108: 442-461.

Received December 21, 2010; accepted in revised form June 3, 2011. 


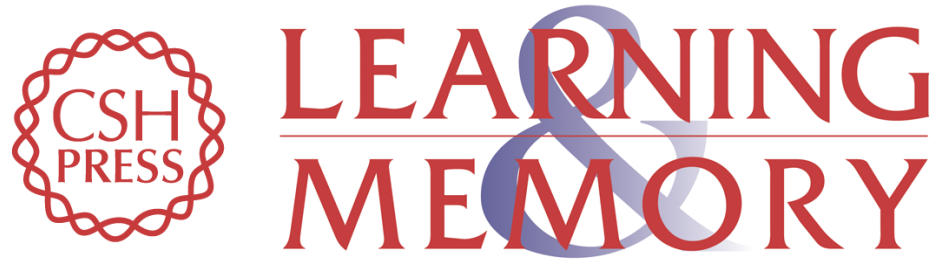

\section{$\beta$-Adrenergic receptors in the insular cortex are differentially involved in aversive vs. incidental context memory formation}

María Isabel Miranda, Elizabeth Sabath, Luis Nuñez-Jaramillo, et al.

Learn. Mem. 2011, 18:

Access the most recent version at doi:10.1101/lm.2126111

References This article cites 34 articles, 8 of which can be accessed free at:

http://learnmem.cshlp.org/content/18/8/502.full.html\#ref-list-1

License

Email Alerting Receive free email alerts when new articles cite this article - sign up in the box at the Service top right corner of the article or click here. 